\title{
Ulcerative Skin Metastasis Lesion in Non-Small Cell Lung Cancer
}

\author{
Jaehyun Bae ${ }^{1}$ (D), Dongkeun Jun ${ }^{1}$ (D), Jeenam Kim $^{1}$ (D), Hyungon Choi ${ }^{1}$ (D), Donghyeok $\operatorname{Shin}^{1}$ (D), Jin Hee Park ${ }^{2}$ (D), \\ Wan-Seop Kim² (D), Myungchul Lee ${ }^{1}$ (D) \\ Departments of ${ }^{1}$ Plastic and Reconstructive Surgery and ${ }^{2}$ Pathology, Konkuk University Medical Center, Seoul, Korea
}

\begin{abstract}
Venous ulcers, ischemic wounds and skin lesions from autoimmune diseases are some examples of unhealing wounds. Practitioners treating such wounds should consider the possibility of skin metastasis of neoplasms, especially in patients with cancer. Treatment of cutaneous metastasis in cancer must include both surgical resection and chemotherapy. Here we present a patient who had lung cancer with skin metastasis. Though incidence of metastasis from lung cancer is known to be as low as $1 \%$ to $12 \%$, its prognosis is poor. Also, the clinical features of these skin lesions tend to vary, often resulting in them being misdiagnosed as benign lesions. The diagnosis of malignancy for this particular case was delayed. After the metastatic lesion was diagnosed as such, surgical resection was performed and the defect caused by wide excision was covered by a superior gluteal artery perforator flap. Though the patient was administered an anticancer drug, the wound healed well after the operation.
\end{abstract}

Keywords: Skin neoplasms; Neoplasm metastasis; Perforator flap

\section{Introduction}

Depending on the type of anticancer drug administered, chemotherapy may delay the healing of a wound [1]. For this reason, chemotherapy for some cancer patients with wounds is postponed until the wound heals, but if the wound was caused by neoplasm metastasis, chemotherapy is actually essential for treatment of the wound. The incidence of cutaneous metastasis among lung cancer patients is low, ranging between $1 \%$ and $12 \%$ [2]. In some reported cases, cutaneous metastasis was the first manifestation of lung cancer before diagnosis of a malignant tumor in the lung [3]. These are some of the reasons why lung cancer patients who have unhealing skin lesions can be misdiagnosed as having only benign lesions.

Cutaneous metastasis derived from lung cancer has a poor prognosis. In the cases of skin metastasis from lung cancer analyzed by Ambrogi et al. [4], the time from diagnosis to death was only up to ten months. Patients therefore need their metastasis to be diagnosed at an early stage and to be surgically treated also with chemotherapy if possible. Practitioners' understanding of the most common sites and clinical aspects of skin metastasis from lung cancer can be helpful in correctly diagnosing the unhealing wound. The most frequent areas of skin metastasis from lung cancer are the chest, abdomen, head, and neck $[3,5]$. It clinically presents as round or oval nodules, relatively firm papules, ulcers, and inflammatory eruptions, and it is painless $[3,5]$.

Here, we report a case involving a lung cancer patient who presented with skin me-

\section{Case Report}

Received: March 17, 2020

Revised: April 13, 2020

Accepted: April 14, 2020

\section{Corresponding author:} Myungchul Lee, M.D.

Department of Plastic and Reconstructive Surgery, Konkuk University Medical Center, 120-1 Neungdong-ro, Gwangjin-gu, Seoul 05030, Korea

Tel: +82-2-2030-8191

Fax: +82-2-2030-5249

E-mail: 20150136@kuh.ac.kr

This is an Open Access article distributed under the terms of the Creative Commons Attribution Non-Commercial License (https://creativecommons.org/licenses/by-nc/4.0/) which permits unrestricted non-commercial use, distribution, and reproduction in any medium, provided the original work is properly cited.

(c) 2020 Korean Wound Management Society 
tastasis. We initially diagnosed it as a simple abscess. However, noting the slow improvement of the wound, we performed a biopsy of the skin lesion for pathological examination and bacteriological culture. Biopsy results confirmed cutaneous metastasis from lung cancer. The study protocol conformed to the ethical guidelines of the Declaration of Helsinki, and a verbal informed consent was obtained from the patient according to the decision of the Institutional Review Board of Konkuk University Medical Center (IRB exemption No. KUMC 202003-017).

\section{Case}

A 67-year-old man with a 10-year history of lung cancer presented to the department of plastic and reconstructive surgery with an inflammatory skin lesion on the left greater trochanter area. He had a variety of chronic medical conditions including diabetes and chronic obstructive pulmonary disease, and had undergone pituitary macroadenoma surgery with testosterone replacement about 11 years ago. The lung cancer was diag-
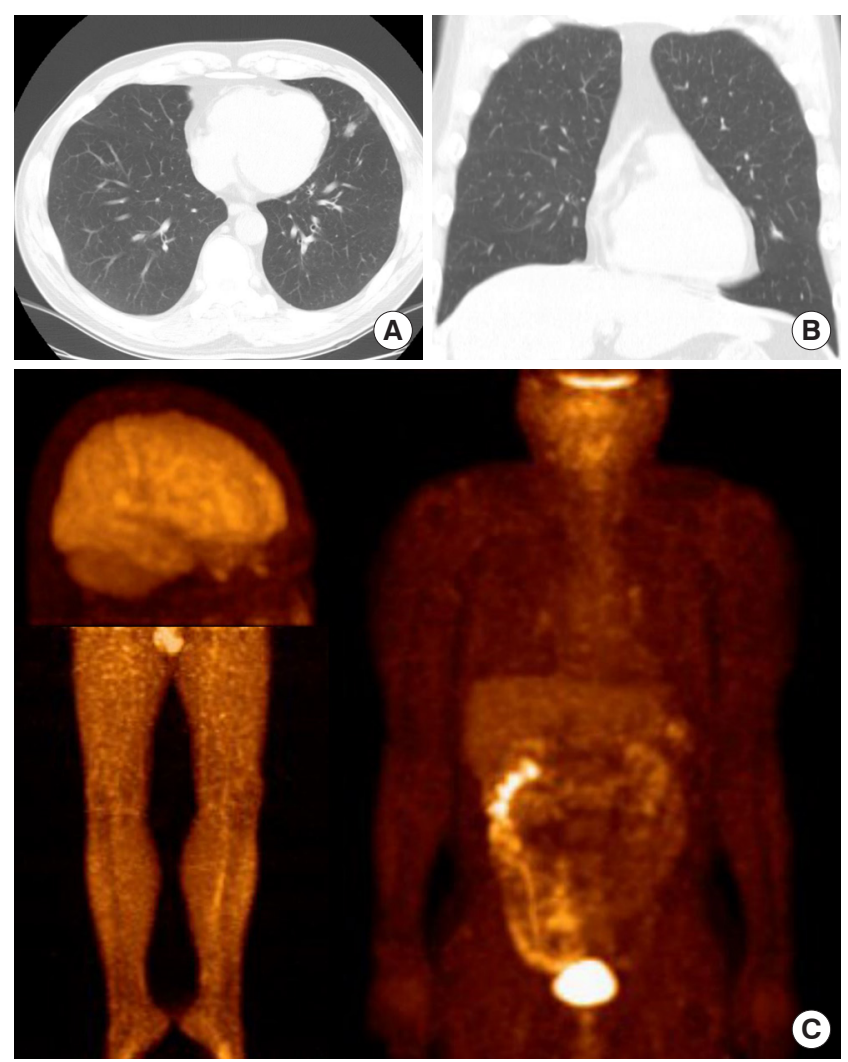

Fig. 1. Imaging study of malignant lesions. The initial computed tomography (A, B) and positron emission tomography-computed tomography $(\mathrm{C})$ of the primary lung cancer. nosed when the patient had a whole body positron emission tomography-computed tomography (PET-CT) and a chest computed tomography (CT) for a regular health checkup 10 years earlier. There was a $1.2 \mathrm{~cm}$ nodular ground glass opacity without a marked increase in ${ }^{18} \mathrm{~F}$-fluorodeoxyglucose (FDG) uptake in the lingula of the left upper lobe of his lung (Fig. 1), but no other abnormal hypermetabolic lesions in the body or brain.

The pathology from lung segmentectomy confirmed bronchioloalveolar carcinoma, one of the lung adenocarcinomas categorized as non-small cell lung cancer (NSCLC) which has no increase in FDG uptake on PET-CT (Fig. 2). The primary lung cancer was stage IA, TNM stage T1N0M0. As molecular pathology showed a K-ras mutation, the patient underwent three cycles of adjuvant chemotherapy with paclitaxel/cisplatin, targeting the K-ras mutation over 2 months. Nine years after the primary lung cancer surgery, the cancer recurred on the left lower lobe and right upper lobe. The patient had a wedge resection of the right upper lobe, followed by chemotherapy with pemetrexed/cisplatin, targeting programmed death ligand 1-expression (22C3) for 1 year.

During the chemotherapy period, 2 months before coming to the department of plastic and reconstructive surgery, he received an intramuscular vitamin supplement injection in the left buttock area at another hospital. Initially, the clinical diagnosis of the inflammatory skin lesion on the left greater trochanter area was an infected epidermal cyst or cellulitis caused

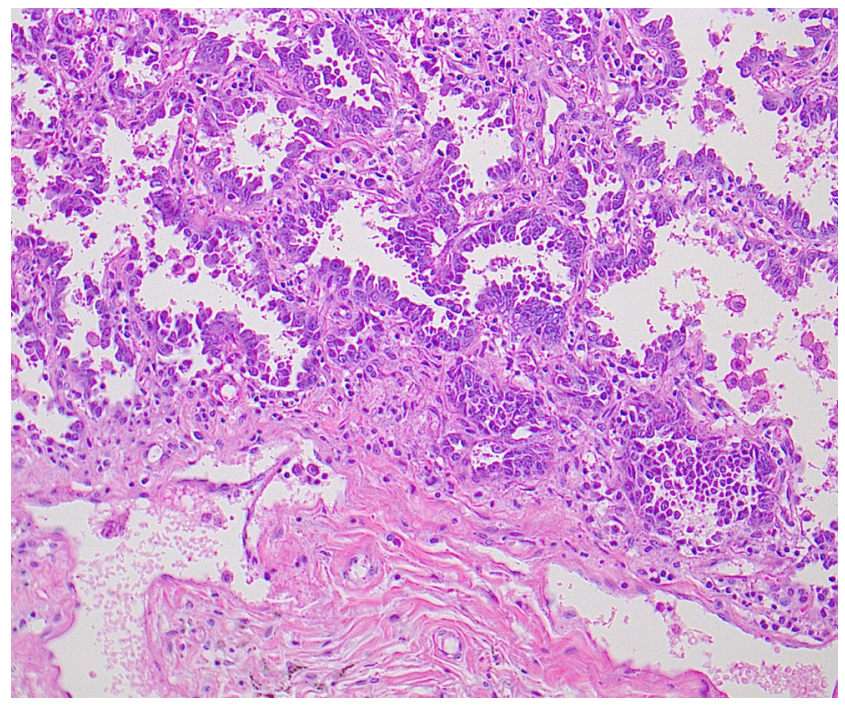

Fig. 2. Pathologic result of primary lung adenocarcinoma (H\&E, $\times 200$ ). 
by the injection. He had incision and drainage of the inflammatory lesion after taking oral antibiotics for a month. The nodule, which was hard on palpation, contained an ulcer measuring $20 \mathrm{~mm}$ at its widest diameter. The hardness of the nodule was considered to be caused by inflammation and scarring of the ulcerative wound, and radiologic studies were not conducted. Despite a month of follow-up care, the wound had not healed (Fig. 3). There was no pus-like discharge, and only minimal bloody fluid.

For management of the lung cancer, we consulted with the pulmonary and respiratory medicine department. As per their
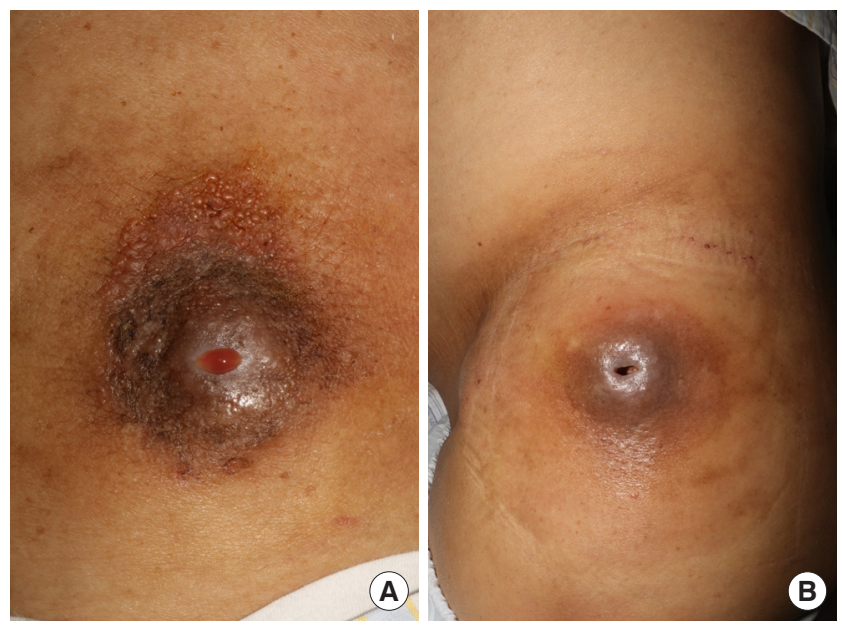

Fig. 3. The initial wound. (A) The small opening presented serous discharge. (B) The ulcerative wound did not heal for 1 month. recommendation, we postponed his chemotherapy to let the wound heal and decided to admit the patient to the department of plastic and reconstructive surgery for observation. Infection markers on initial presentation were as follows: white blood cell count, $12.07 \times 10^{3} / \mu \mathrm{L}$; erythrocyte sedimentation rate, $17 \mathrm{~mm} / \mathrm{hr}$; and C-reactive protein, $0.14 \mathrm{mg} / \mathrm{dL}$. The markers of wound healing were as follows: hemoglobin $(\mathrm{Hb})$ Alc, 7.2\%; Hb, $12.6 \mathrm{~g} / \mathrm{dL}$; and albumin, $3.9 \mathrm{~g} / \mathrm{L}$. There were no identified bacteria in the wound culture. We started intravenous antibiotics (vancomycin $1 \mathrm{~g}$ twice a day) in consultation with the infectious medicine department. We also carried out daily dressing changes to check for signs of infection, including turbid discharge and fever.

After 2 weeks of inpatient treatment, the wound had not improved. An excisional biopsy and tissue culture were performed to check for nontuberculous mycobacteria or other infections. The pathologic findings, which were positive for thyroid transcription factor-1 (TTF-1) and cytokeratin (CK)-7, and negative for CK-20, suggested that the origin of the tissue was the lung. Histologically, TTF-1 is positive in up to $95 \%$ of primary lung adenocarcinomas, so its expression has been used for prognosis prediction in NSCLC (Fig. 4) [6]. The results indicated that the lesion was the same cell type as the primary lung adenocarcinoma. Further, molecular pathology indicated that the metastatic lesion was also positive for the Kras mutation. Although the skin metastasis had a partial sarcomatoid change, features of adenocarcinoma such as glandular formation were still present, as seen in the pathologic results of
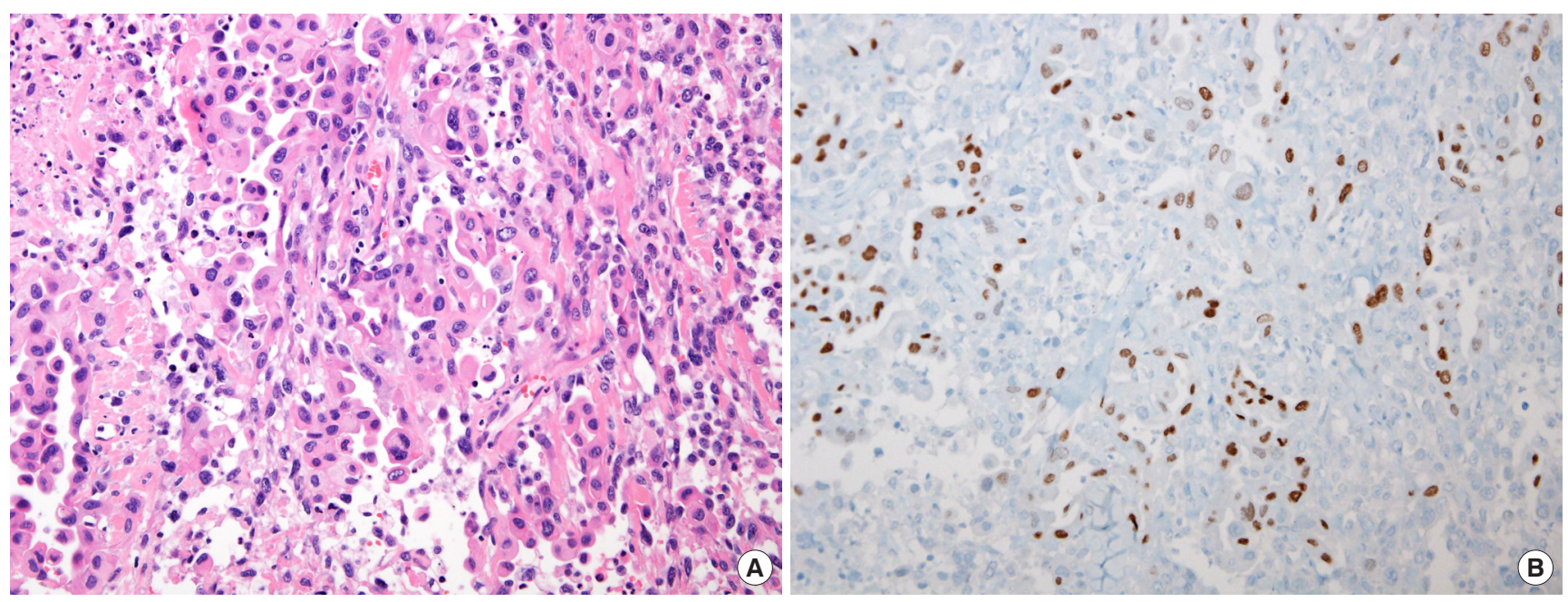

Fig. 4. Pathologic results. (A) Metastatic non-small cell lung carcinoma with some spindle cells. The tumor consists of solid, large epithelial cells with abundant cytoplasm and prominent nucleoli (H\&E, $\times 200)$. (B) Metastatic lung adenocarcinoma is strongly positive for thyroid transcription factor-1 (immunohistochemistry, $\times 200$ ). 
the primary lung cancer (Figs. 2 and 4A).

In consultation with oncologists, we conducted a whole body PET-CT to confirm the presence of other metastasis. Whole body PET-CT compared to the results of a PET-CT 1 year earlier showed a new hypermetabolic mass-like lesion corresponding to the skin lesion on the left greater trochanter area (Fig. 5).

We planned wide excision of the metastatic adenocarcino- ma on the left greater trochanter area. The total size of the lesion requiring resection was about $10 \times 8.5 \mathrm{~cm}$ including a suspected cancer-free margin of $2 \mathrm{~cm}$. We performed wide excision of the lesion, and confirmed on frozen biopsy that the margin of excision was free from cancer (Fig. 6). The wound after excision was fascia depth. Using Doppler ultrasonography, we located some perforators on the right side of the wound margin, and designed an $11.5 \times 7.5 \mathrm{~cm}$ elliptic superior
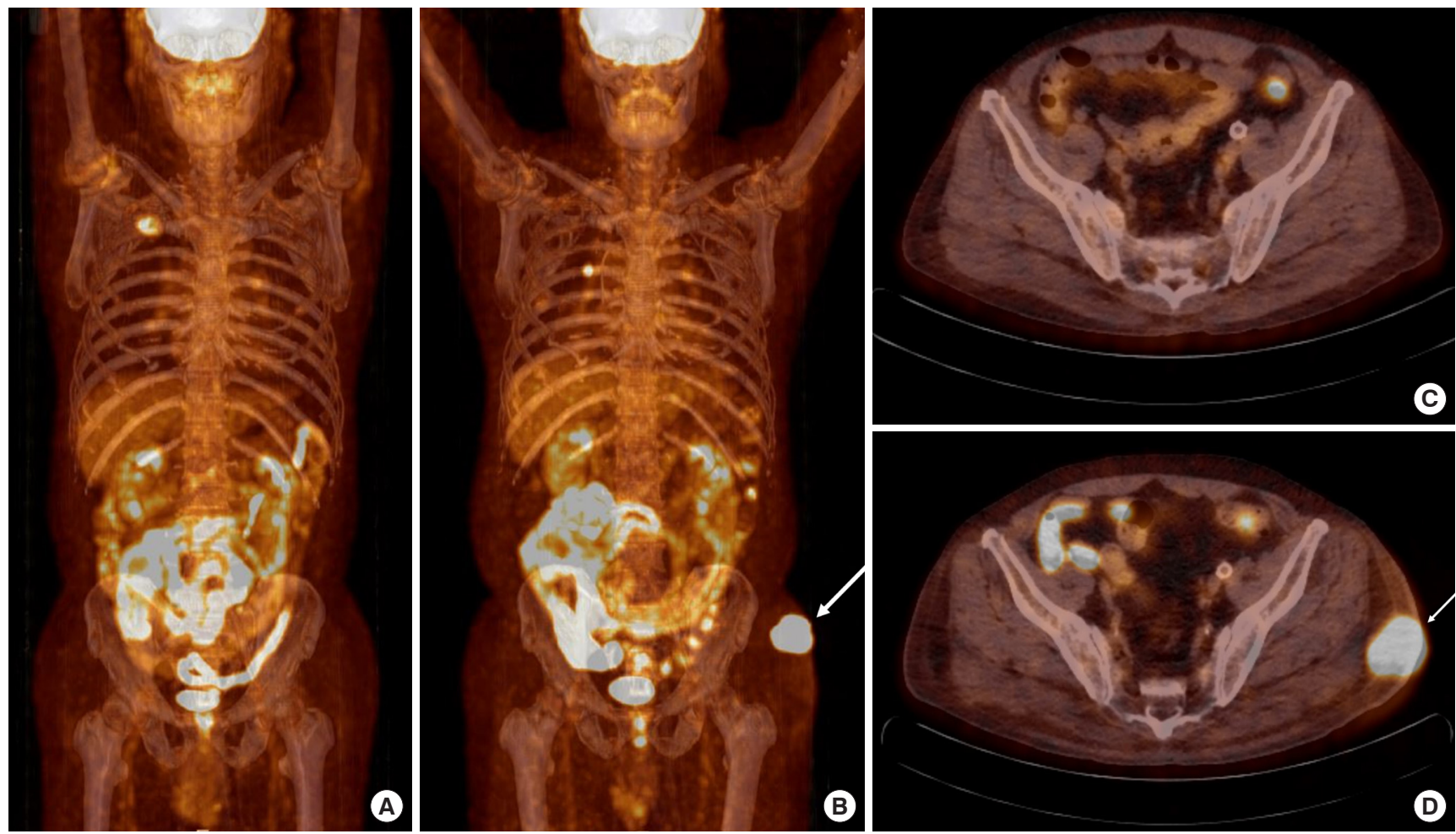

Fig. 5. Imaging studies of metastatic lesion. Coronal view of positron emission tomography-computed tomography (PET-CT) 1-year prior (A) and preoperative (B). Axial view of PET-CT 1-year prior (C) and preoperative (D). Note the suspected metastatic lesion in the left greater trochanter area (white arrows).
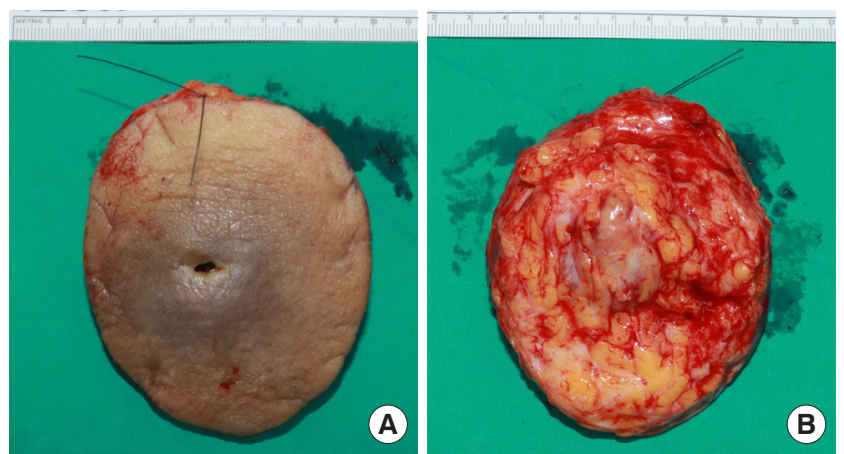

Fig. 6. Surgical specimen for pathologic diagnosis. (A, B) A mass of about $10 \times 8.5 \mathrm{~cm}$ was excised, including a normal margin of $2 \mathrm{~cm}$.
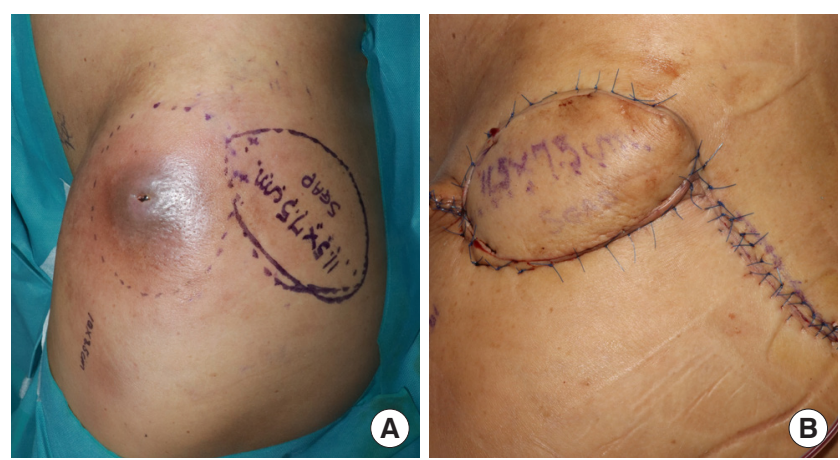

Fig. 7. Operative procedures. Intraoperative photograph (A) and immediately postoperative photograph (B) of the wound after superior gluteal artery perforator (SGAP) flap coverage. 


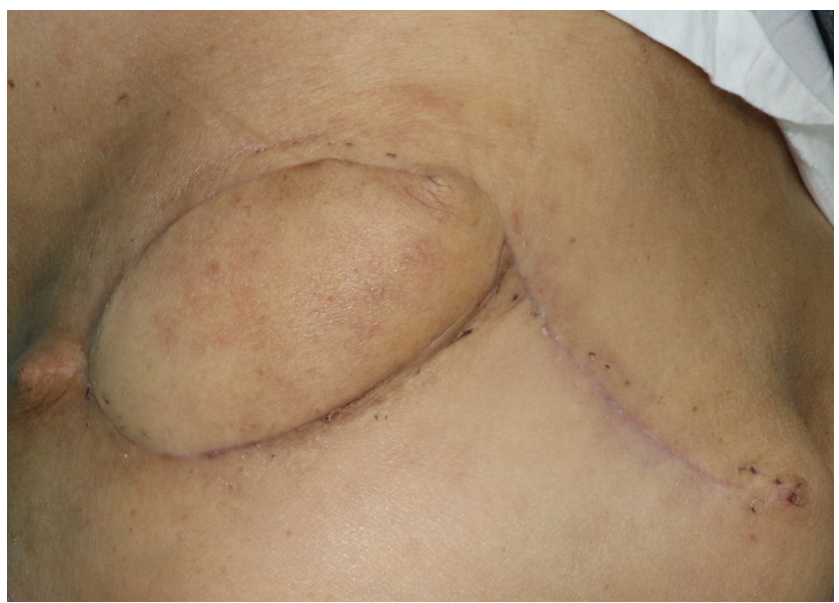

Fig. 8. The healed wound postoperative 3 months.

gluteal artery perforator (SGAP) flap for reconstruction of the soft tissue coverage (Fig. 7).

The patient was referred to the pulmonary and respiratory medicine department for proper management of the stage IVA lung cancer, and palliative chemotherapy was planned with lambrolizumab every 3 weeks starting 10 days after surgery. He underwent the chemotherapy and was discharged home in stable condition. During 3 months of outpatient treatment, he underwent four cycles of lambrolizumab and complete healing of the wound was observed (Fig. 8). As the oncologists evaluated the tumor response as a partial remission, they decided to continue the chemotherapy.

\section{Discussion}

Lung cancer can metastasize to most organs, but mainly spreads to internal organs such as the hilar nodes, liver, adrenal glands, bones, and brain [3]. Metastasis to skin is rare with an incidence of about $1 \%$ to $12 \%$ of all neoplasms [7]. However, some studies report that lung cancer has a high tendency to metastasize in the skin [8]. According to some data, skin metastasis in male patients is highest (24\%) in those with lung cancer compared to other malignancies [9]. In female patients, lung cancer ranks fourth (4\%) for incidence of skin metastasis, following breast cancer, colorectal cancer, melanoma and ovarian cancer [9]. Also, some studies show that adenocarcinoma is the most common type of lung cancer that metastasizes to the skin [3]. In addition, metastatic skin lesions present before or at the same time as the diagnosis of the primary tumor in $20 \%$ to $60 \%$ of reported cases [2].

In this case, the time between the diagnosis of the primary tumor and the discovery of cutaneous metastasis was about ten years, which was quite a long interval compared with previous studies whose mean times were 16.3 and 14.2 months (range, 2-55 months) [7,10]. We hypothesized that the inflammatory reaction of the prior intramuscular injection had an impact on the metastasis. Inflammatory responses are important in tumor formation as the tumor cells metastasize through their release, migration and proliferation [11].

Manifestation of lung cancer metastasis can resemble many benign skin changes, including macules, papules, nodules, ulcerated lesions, and a variety of hyperkeratotic and sclerotic lesions [12]. Furthermore, metastatic cutaneous lesions originating from lung cancer can present in the form of various nodules covered with normal or inflamed skin. The most common type of cutaneous metastasis is nodular, firm, nontender, mobile, and not ulcerated, and the size varies widely from millimeters to centimeters [8]. The area where metastasis takes place most frequently is the thorax, followed by the abdomen, head, and neck [13]. Dhambri et al. [8] said that the cutaneous location was almost ipsilateral to the primary tumor in $90.90 \%$ of cases. Our case was also ipsilateral to the previous lung cancer which was located in the left upper lobe.

Some radiologic studies can help accelerate the diagnosis. Ultrasound can easily detect malignancy in many cases of cutaneous metastasis, but very few ultrasound studies of cutaneous metastasis have been published [12]. PET-CT is important for treating cancer metastasis as it assists identification of malignancies of unknown origin, granted that the lesions are larger than $1 \mathrm{~cm}$. We also found a hypermetabolic lesion on the left greater trochanter area of the patient by performing PET-CT. These imaging studies can help approximate the depth of tumor cell invasion, and whether there is any involvement of nearby muscle or bone. If the result in our case had shown metastasis to muscle or bone, the chosen method could have been free flap for coverage or only conservative therapy.

In this case, we initially planned to postpone chemotherapy until complete wound healing, because we thought chemotherapy might delay the healing of the wound. The mechanism of chemotherapy is to inhibit cell growth including metabolism, division and angiogenesis, and also it blocks the pathways of effective wound repair [1]. Many chemotherapeutic drugs interrupt inflammatory response and block angiogenesis [1]. In addition, some of them have an effect on cell migration to the wound and reduce production of extracellular matrix and collagen by inhibiting fibroblast proliferation [1].

Nevertheless, we decided to remove the skin lesion surgical- 
ly and request immediate chemotherapy intervention when the pathologic results indicated a metastatic skin lesion, because skin metastasis from lung cancer is an unusual event carrying an ominous prognosis and short survival [3]. Lung cancer is known to be the neoplasm that shows the most rapid metastasis to the skin after diagnosis with a mean time of 5.75 months [2]. Some studies report that survival time in lung cancer is about 5 to 6 months after the skin metastasis; the involvement of the skin means that the neoplasm is circulating systemically and developing aggressively [2]. Though solitary skin metastasis is usually treated by surgical resection with chemotherapy, there have been no randomized trials to determine the optimal type of therapy. Other treatments with potential include radiotherapy, radiofrequency ablation, and interferon-alpha injections [14].

While the size of the resection margin is important in preventing the recurrence of cancer recurrence, the appropriate size of the cancer-free margin varies from case to case. In the event of cutaneous metastasis from lung cancer, there is no general consensus on the most appropriate margin for optimal treatment of the skin lesion. In this particular case, we excised $2 \mathrm{~cm}$-margins on the assumption that the lesion was sarcoma. As a result, a $10 \times 8.5 \mathrm{~cm}$ defect on the lateral left buttock required coverage with perforator techniques. As coverage of sacral and trochanteric areas is typically performed with SGAP flap, we located some pedicles to reach the defect and designed an $11.5 \times 7.5 \mathrm{~cm}$ elliptic fasciocutaneous flap. The flap was rotated on the axis of the anterior border of the left gluteus maximus muscle, enabling it to sufficiently cover the trochanteric wound. Bone padding of the iliac crest is crucial to reconstruction in the trochanteric area. A fasciocutaneous flap is quite simple and can preserve anatomical structures and vascularity in case of future operations such as ischial pressure sore reconstruction.

Although the pathologic report in this case indicated that the margins were negative, recurrences requiring additional chemotherapy or radiotherapy are common, as was the case with this patient. The injected anticancer drug administered to the patient, lambrolizumab, is one of the targeted therapies that inhibit immune checkpoints and block tumor cell progression [15]. Recently, targeted drugs have become more widely used in cancer treatment. Targets of some agents include molecules also present in the skin such as epidermal growth factor receptor and vascular endothelial growth factor [1]. For these reasons, Deptula et al. [1] recommend that surgery should be performed 6 to 8 weeks after the targeted ther- apy, as this treatment should not be administered until at least 4 weeks after surgery. In this case, the wound was well healed after wide excision and SGAP flap coverage, though the patient was administered anticancer drugs 10 days postoperative. In addition, the function of the surgical site was preserved well, so the patient was able to walk without any assistive devices.

In conclusion, our experience suggests that general physicians or dermatologists, who are often the first doctors to consult with patients with skin lesions, should consider the possibility of skin metastasis. Physicians should carefully assess the clinical manifestations and medical history of the patient, and perform a biopsy of the lesion to rule out the possibility of metastasis, especially in smokers or in patients with lung cancer. This approach can prevent a delay in treatment of the primary malignant disease and increase survival rates.

\section{Conflict of interest}

No potential conflicts of interest relevant to this article are reported.

\section{ORCID iDs}

Jaehyun Bae https://orcid.org/0000-0001-8716-3109

Dongkeun Jun https://orcid.org/0000-0001-9017-9929

Jeenam Kim https://orcid.org/0000-0002-4080-6135

Hyungon Choi https://orcid.org/0000-0002-3816-1286

Donghyeok Shin https://orcid.org/0000-0002-8450-4411

Jin Hee Park https://orcid.org/0000-0003-1277-7549

Wan-Seop Kim https://orcid.org/0000-0001-7704-5942 Myungchul Lee https://orcid.org/0000-0002-9721-0092

\section{References}

1. Deptula M, Zielinski J, Wardowska A, et al. Wound healing complications in oncological patients: perspectives for cellular therapy. Postepy Dermatol Alergol 2019;36:139-46.

2. Mollet TW, Garcia CA, Koester G. Skin metastases from lung cancer. Dermatol Online J 2009;15:1.

3. Pajaziti L, Hapciu SR, Dobruna S, et al. Skin metastases from lung cancer: a case report. BMC Res Notes 2015;8: 139.

4. Ambrogi V, Nofroni I, Tonini G, et al. Skin metastases in lung cancer: analysis of a 10-year experience. Oncol Rep 2001;8:57-61. 
5. Habermehl G, Ko J. Cutaneous metastases: a review and diagnostic approach to tumors of unknown origin. Arch Pathol Lab Med 2019;143:943-57.

6. Kim JH, Kim HS, Kim BJ, et al. Prognostic impact of TTF1 expression in non-squamous non-small-cell lung cancer: a meta-analysis. J Cancer 2018;9:4279-86.

7. Lee JH, Ahn SJ, Kim HJ, et al. Cutaneous metastasis from lung cancer: a single-institution retrospective analysis. Tuberc Respir Dis 2011;70:139-42.

8. Dhambri S, Zendah I, Ayadi-Kaddour A, et al. Cutaneous metastasis of lung carcinoma: a retrospective study of 12 cases. J Eur Acad Dermatol Venereol 2011;25:722-6.

9. Brownstein MH, Helwig EB. Metastatic tumors of the skin. Cancer 1972;29:1298-307.

10. Marcoval J, Penin RM, Llatjos R, et al. Cutaneous metastasis from lung cancer: retrospective analysis of 30 patients.
Australas J Dermatol 2012;53:288-90.

11. Arias JI, Aller MA, Arias J. Cancer cell: using inflammation to invade the host. Mol Cancer 2007;6:29.

12. Martinez-Moran C, Echeverria-Garcia B, Khedaoui R, et al. Cutaneous metastasis in a patient with lung cancer. Actas Dermosifiliogr 2018;109:372-4.

13. Gupta V, Bhutani N, Marwah N, et al. Scalp metastasis as an initial presentation of lung adenocarcinoma: a case report and literature review. Int J Surg Case Rep 2017;41:32731.

14. Leong SP, Gershenwald JE, Soong SJ, et al. Cutaneous melanoma: a model to study cancer metastasis. J Surg Oncol 2011;103:538-49.

15. Buque A, Bloy N, Aranda F, et al. Trial watch: immunomodulatory monoclonal antibodies for oncological indications. Oncoimmunology 2015;4:e1008814. 